\title{
A hybrid methodology for secondary fragmentation prediction in cave mines
}

\author{
M. Pierce Itasca Consulting Group, Inc., United States of America \\ D.K. Weatherley Sustainable Minerals Institute, The University of Queensland, Australia
}

T. Kojovic SimSAGe Pty Ltd, Australia

\begin{abstract}
Drawing upon numerical studies, published laboratory results and comminution theory, a new methodology has been developed for the prediction of drawpoint size distributions throughout the operational life of a cave mine. The method is 'hybrid' because it utilises both numerical modelling and empirical comminution relations to track the evolution of caved rock size distributions reporting at drawpoints. In this paper we review the current knowledge on caved rock fragmentation, present new results analysing the micromechanics of shear-induced attrition of granular materials, and demonstrate via back-analysis, the applicability of the hybrid methodology for predicting drawpoint fragment size-distributions in existing caving operations.
\end{abstract}

\section{Introduction}

It has been noted within caving mines that the size of rock fragments reporting to the drawpoints tends to decrease as the cave matures. This process is referred to as secondary fragmentation. This is distinct from primary fragmentation, the process by which an initial intact rock mass is disintegrated through the action of induced stresses or gravity to produce a free flowing assemblage of rock fragments. As caved rock is extracted, zones of moving material (known as isolated movement zones or IMZ) extend above the drawbells. Numerical modelling has shown (Lorig and Cundall, 2000; Pierce, 2004) that within an IMZ, material flows uniformly downwards at low stress whilst on the edge of an IMZ large stresses and shear strains develop. In stagnant regions surrounding IMZs high compaction stresses are likely to develop due to overburden pressure and stress-shedding from nearby movement zones. We postulate that secondary fragmentation is driven by the high stresses and large shear strains within and surrounding movement zones. In the work that follows we develop a methodology to predict the degree of secondary fragmentation taking account for the stress-strain history experienced by caved rock during its transit through a cave.

Secondary fragmentation impacts caving operations in several ways. Two of the more significant impacts are:

- Secondary fragmentation causes the mean fragment diameter to decrease with draw/time. This will result in narrower IMZs. Secondary fragmentation must therefore be taken into account when designing drawpoint layouts to ensure IMZ interactivity, uniform drawdown and optimal recovery throughout the operational life of a cave.

- Secondary fragmentation produces fine material that may travel more quickly down the draw column than coarser fragments. Fines can negatively impact downstream processing efficiency and may also be of a different grade than coarser fragments, causing an impact on grade-tonnage trends.

As a result of the above factors, there is considerable value in providing a means for predicting secondary fragmentation. Dr D.H. Laubscher, in collaboration with A.R. Guest and P.J. Bartlett, developed the program BCF (Block Cave Fragmentation) for prediction of both primary and secondary fragmentation at the Premier Mine, South Africa (Brown, 2003). The program was developed by Dr G.S. Esterhuizen and was improved during 1998 and 1999 as part of the International Caving Study Stage I (Esterhuizen, 1999). BCF uses a simplified technique for determining in situ block sizes (i.e. primary fragmentation) and uses empirical rules to predict how the blocks will subsequently reduce in size within the draw column via secondary 
fragmentation. The secondary fragmentation calculations in BCF are based entirely on rules established through experience and engineering judgment (Esterhuizen, 1998). Experience with the program suggests that some of the assumptions and empirical rules used in the program could be further improved, including those developed for secondary fragmentation (van As, 2006, written comm.). In his review of BCF, Brown (2003) proposed that the mechanics of the problem be studied in more detail and suggested the following way forward:

"The approach suggested for the development of an improved method of predicting secondary fragmentation, is to initially study the basic mechanics of the each of the major mechanisms involved using analytical and numerical models. From these studies and parametric numerical stress analyses, a set of empirical rules for each the mechanisms will be developed. In some cases, the rules used in BCF may provide an appropriate starting point, or indeed, the only practicable option. It is considered that the development of the empirical rules will also benefit from the results of distinct element numerical simulation studies." (Brown, 2003)

This adequately describes the aim of the work described here. In the next section we review current understanding of the physical mechanisms responsible for secondary fragmentation, including a discussion of laboratory, empirical and experiential approaches to secondary fragmentation prediction. In the following section we present results of new numerical studies shedding light on the efficiency of frictional shear work to induce fragmentation within IMZs. Finally we outline a new hybrid approach for the prediction of secondary fragmentation within cave mines, utilising a combination of numerical and empirical techniques. The paper concludes with a discussion of the methodology, its advantages and limitations, and directions for improvement of the methodology.

\section{Review of current knowledge}

In this section we review current knowledge on secondary fragmentation, including experiential results from caving operations (incorporated into BFC), postulated mechanisms for secondary fragmentation and laboratory or empirical approaches to quantify or predict the size-distribution of fragmented materials taking account for factors such as mode and level of loading stresses or strains, and the shape, angularity and strength of particulate materials. On the basis of this review, we conclude that the three main mechanisms for secondary fragmentation in block caves are:

- impact fragmentation when rock blocks fall through an air gap

- shear-induced fragmentation predominantly at the boundary of movement zones

- compression-induced fragmentation within stagnant zones.

\subsection{Review of postulated mechanics for secondary fragmentation}

There are two main modes of fragment breakage that have been postulated to occur within caves (Esterhuizen, 1998; Laubscher, 2000): block splitting and corner rounding. Block splitting refers to the breakage of the entire fragment into two or more pieces whereas corner rounding refers to the removal of small fragments (or fines) from the surface of the block. The review here will demonstrate that the postulated failure modes are consistent with the results of published experiments on the yielding of granular materials such as soils, rockfill and other materials. In the literature, the process of particle breakage is often referred to as attrition and the modes of breakage are referred to as bulk fracture (or crushing) and abrasion.

The yielding of granular materials has been most widely studied under the following test conditions:

- uniaxial compression

- triaxial compression

- isotropic compression

- direct shear.

Based on in situ observations (Laubscher, 2000), the examination of the kinematics and mechanics of flow within PFC3D simulations (Lorig and Cundall, 2000; Pierce, 2004) and the results of physical modelling 
studies (e.g. Castro, 2006) we expect all four conditions to occur within a cave, particularly when isolated draw is occurring. As illustrated in Figure 1, the central plug flow region of a movement zone is characterised by low strains and low stresses, resulting in isotropic compression. The outer shearing region of a movement zone is characterised by low stresses and high shear strains, resulting in conditions similar to direct shear tests. Finally, the stagnant zones are characterised by high stresses and low strains, resulting in one dimensional and triaxial compression. Conditions of high stress and high strain might also exist between drawpoints under the interactive draw mechanisms proposed by Laubscher (2000). In this case, the stress conditions are likely to be triaxial in nature but carried to very high strain levels. For the purposes of discussion and review, we will consider secondary fragmentation under conditions of compression and shear, which broadly characterise conditions in the stagnant and moving zones respectively. We also consider secondary fragmentation via impact loading, which can occur when rock fragments fall through an air gap.

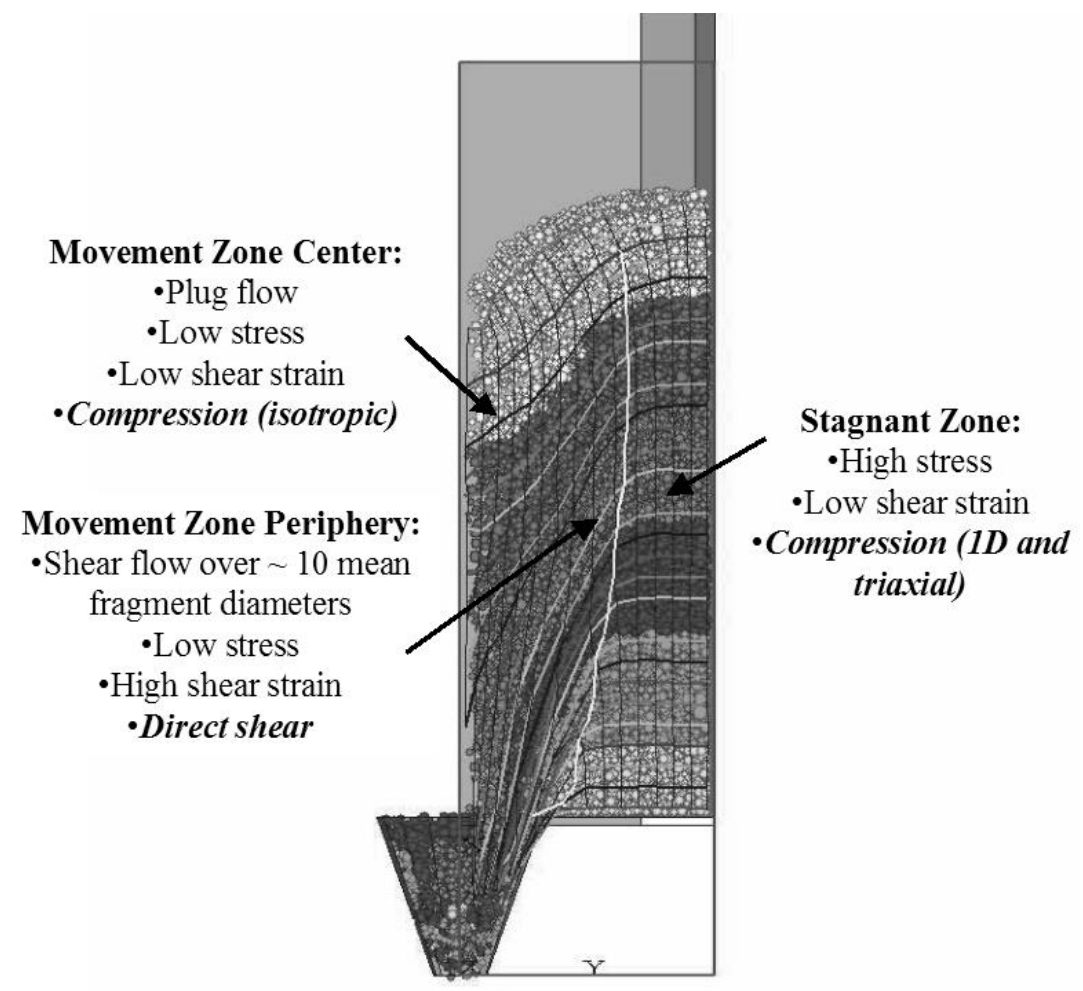

Figure 1 Different stress and strain conditions associated with an isolated movement zone, as obtained from PFC3D quarter-symmetry simulations of gravity flow in block caves (Lorig and Cundall, 2000; Pierce, 2004)

\subsection{Review of published research on fragmentation of granular materials}

We present in this section, the main conclusions from previous laboratory studies on the fragmentation of granular material. We focus upon research aiming to identify the factors controlling the occurrence and degree of fragmentation observed for granular assemblies undergoing compaction, shear and impact. As prediction of the evolution of fragment size-distributions within block caves is our goal, we concentrate upon empirical models that aim to predict the size-distribution of fragmented materials as a function of the factors controlling fragmentation.

\subsubsection{Compression-induced fragmentation}

The results of laboratory studies (Marsal, 1967; Lee and Farhoomand, 1967; Yamamuro and Lade, 1996; Lade and Yamamuro, 1996; Yamamuro et al., 1996; Nakata et al., 2001) and analytical studies (McDowell et al., 1996; McDowell, 2005) have demonstrated that splitting is the primary mode of fragment failure in granular materials subject to compression. Splitting results from tension induced in a fragment via compression or bending. If the induced tensile stress exceeds the tensile strength of the grain, it will split $-\mathrm{a}$ result consistent with the definition of tensile strength of concrete in the Brazilian test. 
The results of single particle crushing tests by Lee (1992) indicate that in addition to splitting, compression of a granular assembly may result in some rounding. Based on the typical form of a load-displacement curve generated by Lee (1992) and McDowell and Bolton (1998) suggest that rounding can occur at low stress values (relative to intact rock strength) followed by splitting at higher stress levels. The postulated mechanism being the fracturing, at low overall stress, of asperities on the surface of the grain, resulting in the rounding as small corners break off the grain.

The results of laboratory compression tests on sand, gravels and rockfills by Lee and Farhoomand (1967), Fukumoto (1992), Yamamuro (1993), Coop et al. (2004) and others indicate that compression results in a rotation of the fragment size-distribution around the largest particle size. In other words, compression results in the generation of smaller fragments via splitting or rounding but the largest fragments tend not to be crushed or split. While this is somewhat counter-intuitive (since larger fragments are generally weaker), McDowell et al. (1996) points out that large fragments also have more contacts with neighbouring fragments; this effectively confines and stabilises them, reducing the induced tensile stress. Smaller fragments, on the other hand, have fewer neighbour contacts and are more likely to split. McDowell et al. (1996) were able to show that this behaviour causes granular materials to evolve toward a fractal size distribution when subject to secondary fragmentation via splitting. Turcotte (1986) and McDowell and Bolton (1998) demonstrate that when materials such as coal, granite and basalt are subject to compression, the gradient of their size distribution is generally around 0.5 , which corresponds to a fractal dimension of 2.5 (Turcotte (1986) provides a detailed explanation of the fractal dimension and how it is derived).

Hardin (1985) examined fragmentation in compression tests on approximately 30 different soils, ranging from rounded quartz sands to angular crushed granite. He was able to study a wide range of parameters, including particle size distribution, particle shape (angularity), stress, porosity and particle strength. As a result, he was able to develop an empirical model for crushing of soil particles that may be relevant to the study of secondary fragmentation via compression in caves. Hardin's (1985) extensive research indicated that in addition to particle size-distribution, fragmentation was impacted by stress, strength, void ratio and angularity as follows:

- fragmentation increases with increasing ratios of stress to fragment strength

- fragmentation increases with increasing void ratio (or porosity) - this is due to the fact that the number of neighbour contacts decreases as the void space increases, resulting in higher induced tensile stresses in fragments

- fragmentation increases with increasing angularity.

Combining all these factors, Hardin (1985) outlines an empirical approach to predict the total breakage of compressed granular materials, however, the methodology does not predict the fragment size-distribution resulting from the compressive load.

Pierce (2007) conducted a numerical study on the simulation of secondary fragmentation under compression using PFC3D. Samples of caved rock, which consist of assemblies of angular fragments represented by clusters of bonded PFC3D particles, were derived from the results of synthetic rock mass (SRM) testing. It has been demonstrated that these SRM tests result in 'synthetic caved rocks' (SCR) with size distributions that match well with the observed primary fragmentation in cave mines (Pierce et al., 2006). By subjecting these synthetic samples of primary fragmented rock to further loading (isotropic and one-dimensional compression), the additional changes in particle size distribution resulting from compression-induced secondary fragmentation could be quantified. The predictions for compression-induced total breakage from Hardin's (1985) model, compared favourably with the results of these PFC3D simulations. Although the PFC3D models currently suffer some restrictions on the minimum size of SCR fragments, the numerical approach offers hope for development of predictive relationships for the size-distribution of fragmented materials under compressive loads.

\subsubsection{Shear-induced fragmentation}

The results of a number of laboratory studies (Paramanathan and Bridgwater, 1983; Ghadiri et al., 2000; Bridgwater et al., 2003; Coop et al., 2004) have demonstrated that under shearing conditions, rounding tends to be the dominant mode of fragment yield at low ratios of normal stress to intact strength while splitting 
becomes more dominant at higher ratios. As will be discussed later, it is likely that the stress:strength ratios possible within an IMZ could result in both modes of failure under shearing.

There is evidence to suggest that during shearing under high stress:strength ratios, the fragment size distribution rotates around the maximum particle size (i.e. the percentage of finer fragments increases but the largest fragments maintain their original size) and evolves toward a stable grading curve that plots as a straight line in log-log space. This evolution toward a fractal distribution is the same as observed during compression (see Section 2.2.1). These trends were observed by Coop et al. (2004) in angular carbonate sands subject to shearing under high stress (where the primary mode of attrition is splitting). When plotted in $\log$ - $\log$ space their stable distribution exhibited a gradient of 0.43 , which corresponds to a fractal dimension of 2.57; this is close to the value of 2.5 generally observed during compression.

The size distribution of a granular material appears to evolve in a different manner when subject to shearing at low stress:strength ratios. In this case, rounding is the dominant mode of failure and so a larger proportion of fines are created. Bridgwater et al. (2003) demonstrate that for an initially mono-sized material, a bimodal size distribution results, consisting of very fine material $(<0.2 \mathrm{~mm})$ and large particles within $90 \%$ of their original size.

Bridgwater et al. (2003) examined attrition via shearing under a wide range of normal stress, shear strain, particle shape and particle strength. Bridgwater et al. (2003) asserts that the mechanics of attrition for initially mono-sized assemblies, when evaluated over a broad range of stresses and strains, may be united in a simple manner, by way of an empirical attrition law:

$$
W=K_{N}\left(\frac{\sigma_{N} \Gamma^{\phi}}{\sigma_{s c s}}\right)^{\beta}
$$

where:

$$
\begin{array}{lll}
W & = & \text { the mass fraction attrited from the mono-sized initial assemblies (per cent). } \\
\sigma_{N} & = & \text { the normal stress applied to the assembly (MPa). } \\
\Gamma & = & \text { the total shear strain applied to the assembly (dimensionless). } \\
\sigma_{s c s} & =\quad \text { the fracture strength of the constituent particles in the assembly (MPa). } \\
K_{N}, \beta, \phi & =\quad \text { empirical constants (dimensionless). }
\end{array}
$$

The equation indicates that secondary fragmentation under shearing is strongly controlled by the product of stress and shear strain, which is essentially the work done on the material. In addition to Bridgwater et al.'s (2003) empirical model (which may be useful for first-pass estimates of shear-induced secondary fragmentation in cave mines), their raw experimental data was used in a numerical study described in Section 3, to determine the fraction of shear work that results in attrition under low stress conditions, an important input to the our hybrid fragmentation prediction methodology described in Section 4.

\subsubsection{Impact-induced fragmentation}

Numerous published studies have been conducted on impact-induced fragmentation of rock, particularly in the field of minerals processing. A complete review of these works is beyond the scope of this paper, so instead we discuss two empirical comminution models derived from laboratory studies on impact breakage of rock and rock-like materials.

Vogel and Peukert $(2003,2004)$ recently published two papers on the subject of a model describing breakage probability in relation to material properties and a method to determine model parameters. Their model was developed based on a theoretical approach, considering two different procedures: a generalised dimensional analysis approach proposed by Rumpf (1973); as well as a detailed statistical fracture mechanical model based on Weibull (1951). In this model, the probability that a particle will break due to impact is determined by the product of initial particle size, the total mass-specific net impact energy and a material breakage parameter which can be measured via laboratory experiments involving single particle impact (e.g. the Julius Kruttschnitt Mineral Research Centre (JKMRC) Drop Weight testing device described below). In much the 
same way as Bridgwater et al.'s (2003) model, the Vogel and Peukert relation offers promise as a way to quickly estimate whether significant impact-induced fragmentation will occur if air gaps develop within an operational cave.

In JKMRC ore breakage characterisation, breakage (or appearance) functions are determined from single particle breakage tests using a drop-weight testing device (Napier-Munn et al., 1996). This device is configured to determine the product size obtained from breaking particles of particular initial sizes, at a range of input energies. The product size distributions are described by the t-family of curves, which are a convenient and compact way of representing the breakage function. The principal breakage parameter which is used to characterise the t-curves is the $t_{10}$, which is defined as the cumulative percentage of broken particles smaller than one tenth of the geometric mean size $(\mathrm{Y})$ of the test particle. This parameter is uniquely related to other points on the size distribution curve; $t_{2}, t_{4}, t_{25}, t_{50}$ and $t_{75}$ for a given ore type. Knowing the curves for a particular material, and given a $t_{10}$ (from a given Ecs, or from a model), the full product size distribution can then be reconstructed. The degree of breakage, or breakage index, $t_{10}$, is related to the specific comminution energy (or comminution energy per unit mass) as follows:

$$
t_{10}=A\left(1-e^{-b E_{c s}}\right)
$$

where:
$A, b=$
the empirical ore impact breakage parameters.
$E_{c s} \quad=$
the specific comminution energy $\left(\mathrm{J} \mathrm{Kg}^{-1}\right)$.

A steeper gradient of the $\mathrm{t}_{10}-E_{c s}$ curve indicates a softer ore. For a constant value of A, this translates into a higher value of $b$. The $t_{10}$ can be interpreted as a 'fineness index' with larger values of $t_{10}$ indicating a finer product size distribution. In crusher breakage $t_{10}$ is typically around $3-10 \%$ for primary, $10-15 \%$ for secondary and $15-25 \%$ for tertiary crushing. The value of parameter $\mathrm{A}$ is the limiting value of $\mathrm{t}_{10}$. This limit indicates that at higher energies little additional size reduction occurs as the Ecs is increased, i.e. the size reduction process becomes less efficient.

One distinct advantage of the JKMRC $\mathrm{t}_{10}$ comminution model over the over empirical breakage models described above, is that it predicts the entire size-distribution of fragments arising from an impact of a given comminution energy $\left(E_{c s}\right)$. Hardin's (1985) model predicts only the total compression-induced breakage as a percent, Bridgwater et al.'s (2003) model predicts only the fraction of material abraded during shear, and Vogel and Peukert's (2004) model only predicts the probability of breakage as the result of impact. It is for this reason that we have adapted the $\mathrm{t}_{10}$ comminution model for the prediction of secondary fragmentation in the hybrid method described in Section 4. To adapt this comminution model, it is necessary to determine physically reasonable ways to estimate the equivalent comminution energy for shear-induced and compression-induced secondary fragmentation. In the following section, we describe a numerical study involving PFC3D and the raw data from the Bridgwater attrition experiments (Bridgwater et al., 2003) in order to derive a relationship for estimating the equivalent comminution energy for fragmentation due to shear. In future investigations we will further derive equivalent comminution energy for compressioninduced secondary fragmentation.

\section{$3 \quad$ Numerical studies on the comminution efficiency of frictional shear work}

The laboratory results of Bridgwater et al. (2003) demonstrate that extrudate particles undergo both rounding and splitting. Splitting may be incorporated in PFC3D by replacing individual particles with an ensemble of smaller particles when given stress conditions are exceeded. This is a computationally efficient approach, minimising the number of particles to be simulated, however, care must be taken to ensure mass and momentum are conserved when particles fragment. To directly simulate the attrition of fine materials from the surface of macro-particles, aggregates or clusters of particles flow code (PFC) particles might be used. This approach is considered computationally intractable at present (due to the large number of particles required) so an alternative methodology has been developed; namely, to incorporate a microphysical attrition mechanism as described in the following section. The basic concept is that individual particles are initially assigned an amount of attritable fine material that is progressively worn away during frictional interactions 
between PFC particles, the rate of attrition being proportional to the incremental slip work imparted to each particle.

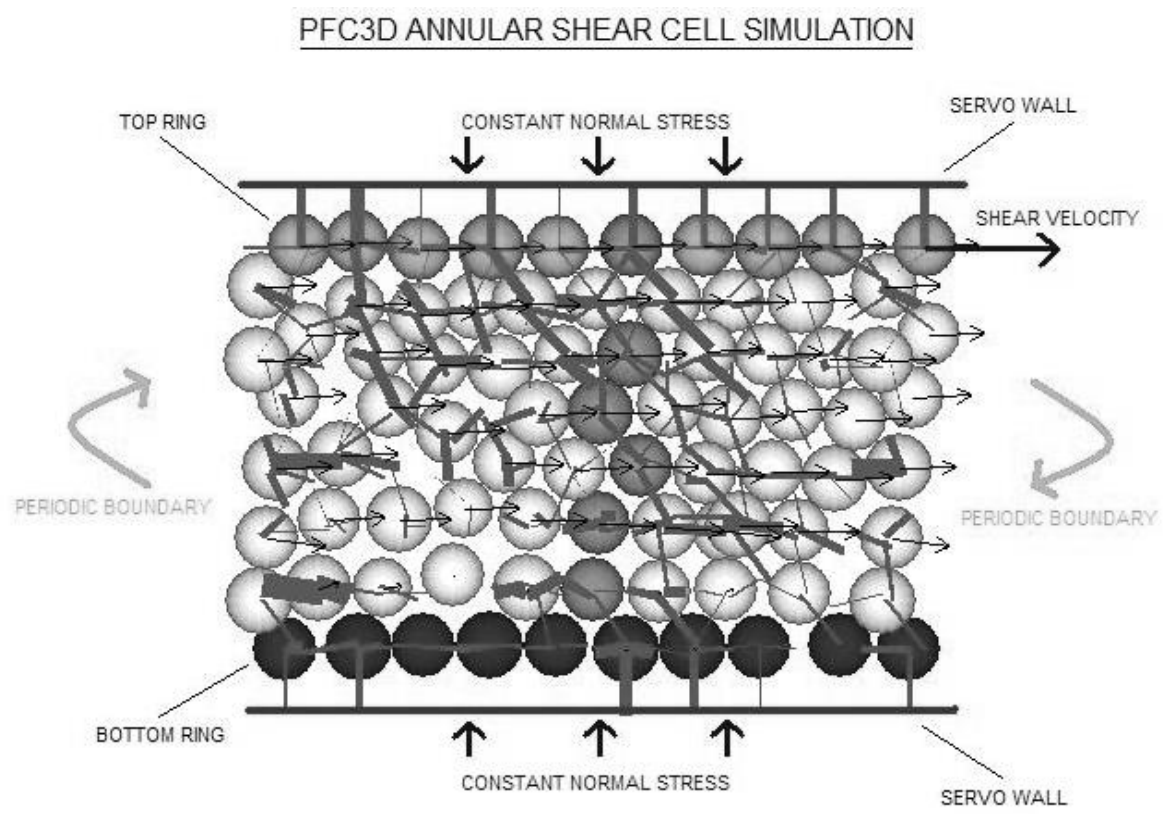

Figure 2 Illustration of the PFC3D annular shear cell model utilised to study attrition of granular materials under shear

\subsection{Model geometry}

The PFC3D model for the annular shear cell (Figure 2) consists of a block of unbonded PFC particles sandwiched between two rough plates constructed from thin plates of particles joined into a clump. The top plate represents the top ring of the annular shear cell and is driven at constant speed in the horizontal shear direction. It is attached to a servo wall that maintains a constant normal stress throughout the simulation. The bottom plate (representing the bottom ring) remains stationary. We employ periodic boundaries in the shear direction so that particles leaving the model region to the right re-enter on the left side of the region. Use of periodic boundaries permits simulations of very large total strain within a relatively small model. Figure 3 shows the schematic of Bridgwater's annular shear cell (Bridgwater et al., 2003) modelled using PFC3D.

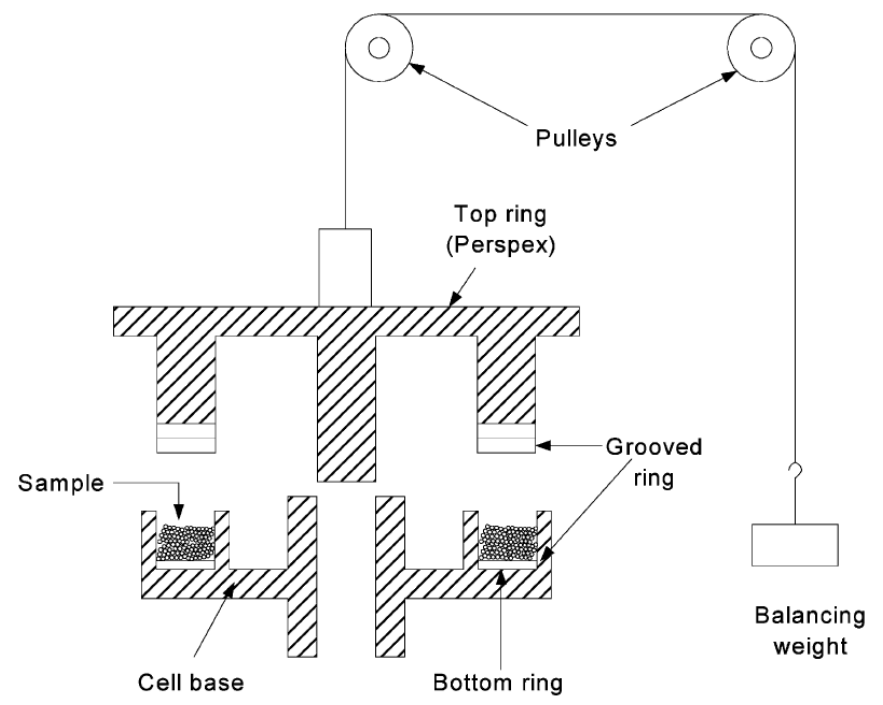

Figure 3 Schematic of the Bridgwater annular shear cell (Bridgwater et al., 2003) 


\subsection{Microphysical attrition law}

Each PFC particle $(i)$ is assigned a physical property, $M_{i}(t)$ representing the remaining volume of attritable material at a specified time $t$. Physically, $M$ represents the fraction of attritable mass (or surface asperities) that remains attached to (or carried by) the PFC particle. Initially we set $M_{i}(0)=1$ signifying that the particle carries all of its attritable mass.

We postulate that the rate at which attritable mass is removed from a particle, during frictional interactions with other particles, is proportional to the frictional slip work, $W_{\text {slip }}$ and introduce a dimensionless parameter $\xi<1$ representing the efficiency with which slip work attrites mass from the particle. We further postulate that the amount of attrition is proportional to the remaining attritable mass. In other words, more heavily attrited particles lose less mass per unit slip work than less heavily attrited particles. This represents the physical observation that particles become more rounded when exposed to larger strains and hence become less susceptible to attrition.

Let $W_{i c}(t)$ be the frictional slip work during the previous time step $(\Delta t)$ experienced by particle $i$ due to a frictional contact $c$ with a neighbouring particle. Then the attritable mass fraction is updated according to the relation:

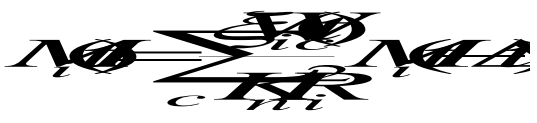

where:
$K_{n} \quad=$
the normal contact stiffness between particles $(\mathrm{N} / \mathrm{m})$.
$R_{i} \quad=$
the radius of particle $i(\mathrm{~m})$.

If the frictional slip work is assumed to be a constant, a discrete recurrence relation of this form results in an exponential decay in the attritable mass fraction with time. It is reasonable to expect that the ensemble average $\langle\mathrm{M}\rangle(\mathrm{t})$ of the attritable masses should approximate an exponential in the limit of large strain; one expects all particles to be subjected to approximately equal frictional slip work on average. Consequently, for the PFC3D annular shear cell experiments (in which strain increases linearly with time), we expect the ensemble average attritable mass to approximate:

$$
\langle M\rangle(\Gamma)=A e^{-(\xi \hat{\omega} \Gamma)}
$$

where:

$$
\begin{array}{lll}
\Gamma & = & \text { the total shear strain. } \\
\hat{\omega} & = & \text { the average frictional slip work per particle. }
\end{array}
$$

In the results that follow, this relation will be fitted to numerical time-series of the ensemble average attritable mass fraction obtained from PFC3D simulations with varying normal stresses applied to the servo walls.

\subsection{Analysis of Bridgwater et al. (2003) data versus numerical results}

Two parameter studies have been undertaken to examine the effectiveness of the microphysical attrition law in reproducing the results of Bridgwater et al. (2003): one in which the attrition parameter is varied and another in which the normal stress is varied.

The first parameter study involved four simulations with a constant normal stress of $24.8 \mathrm{kPa}$ and differing values of the attrition parameter $\xi \in\{0.001,0.01,0.1,1.0\}$. This initial test permits validation of the assertion (Equation (4)) that the ensemble average attritable mass should decay exponentially with the decay rate proportional to the attrition parameter. The results are illustrated in Figure 4. The equivalent results for Bridgwater et al.'s (2003) extrudate 'C' experiment are provided for comparison. In detail, we compute the fraction of mass that does not pass the largest sieve size $(2,800 \mu \mathrm{m})$ as the experimental equivalent of the ensemble average attritable mass fraction. 
The results show that the mean frictional slip work is independent of the attrition parameter for the smaller three values of $\xi$ with a quality least-squares fit of the attrition data to an exponential decay. The fit is considerably worse for the end-member case $(\xi=1)$ which is expected given that one would not suppose that all of the frictional slip work is expended on attrition of the particles. Numerically, the deviations from an exponential arise due to unrealistically large (or near total) attrition of a subset of the particles during relatively isolated shear events. Thereafter, the attrition of these particles contributes little towards the evolution of the attritable mass fraction of the ensemble.

An estimate for the mean frictional slip work per particle may be obtained from the results of the first three simulations $\langle\hat{\omega}\rangle \approx 0.03245 \pm 0.00027$. Utilising this value one may estimate the value for the attrition parameter that would yield identical results as the laboratory experiment i.e.:

$$
\xi_{B}=\frac{\alpha_{B}}{\langle\hat{\omega}\rangle} \approx 0.04650 \pm 0.00027
$$

In other words, the attrition results of the laboratory experiment may be obtained via the microphysical attrition law assuming that approximately $4.7 \%$ of the frictional slip work is expended on attrition; a result that is not beyond physical reason.

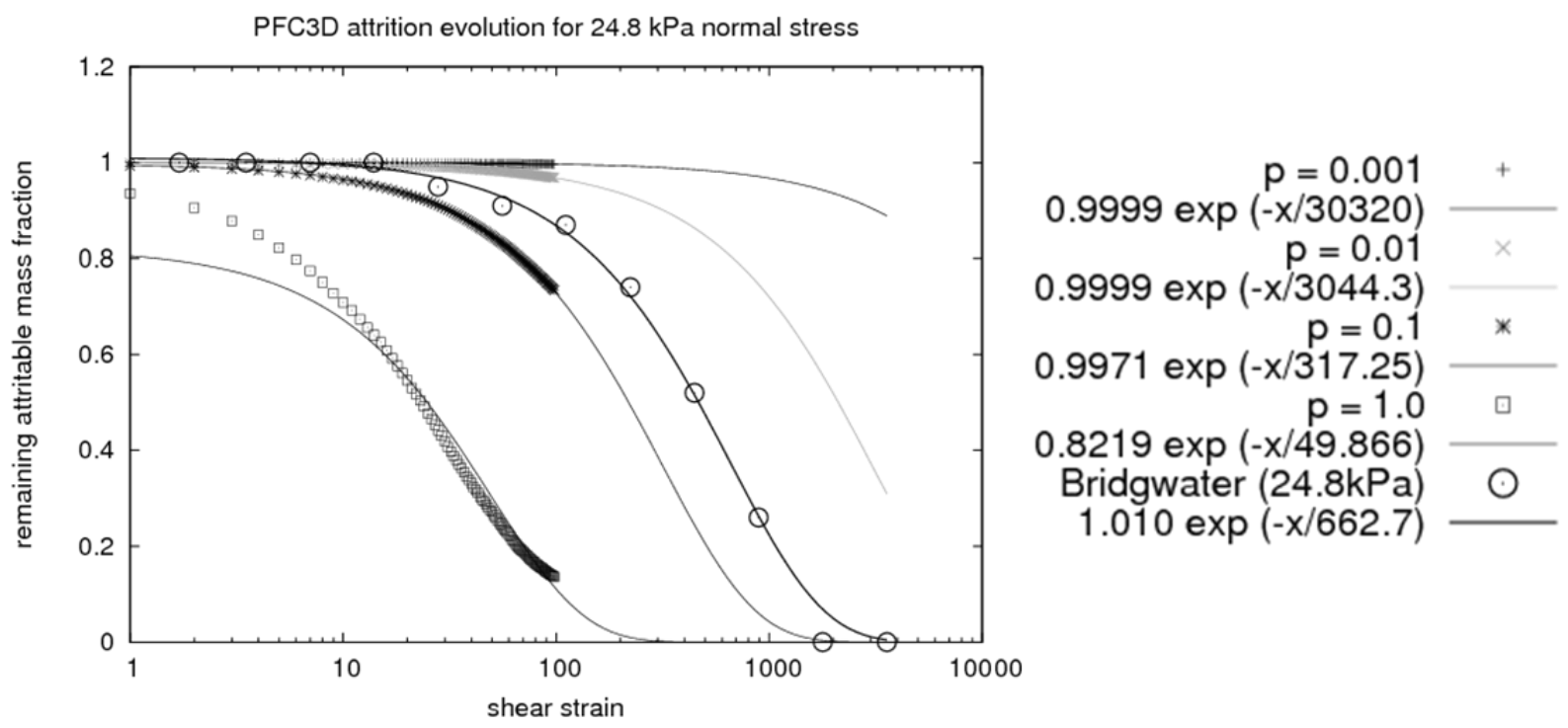

Figure 4 PFC3D attrition evolution versus shear strain for a parameter study in which the attrition parameter $(\xi)$ is varied for constant normal stress $\sigma_{N}=24.8 \mathrm{kPa}$. For comparison, the equivalent laboratory results from Bridgwater et al.'s (2003) extrudate ' $C$ ' experiments are plotted as black circles with the corresponding exponential fit to the data

The second parameter study focuses on the effect of varying the normal stress applied to the PFC3D shear cell apparatus. Initially there was some debate as to whether the microphysical attrition law would be capable of reproducing the laboratory results under differing normal loads, given the renormalisation of frictional slip work in the attrition rate equation. It should be noted that an increased external load will be transmitted to individual particle contacts, resulting in a higher (on average) frictional slip work per particle contact per unit time. Thus one would expect that the decay rate of the attritable mass fraction will display stress dependence.

To examine this dependence, we simulated the shear cell experiment for a constant attrition efficiency of $\xi=0.1$ and normal stresses matching those of the laboratory experiment for extrudate ' $\mathrm{C}$ ' and additional values in the range $24.8-150 \mathrm{kPa}$. Once again we compute the ensemble average attritable mass fraction and fit the time-series to an exponential decay relation. One may again back-calculate the value for the attrition parameter that would theoretically reproduce the laboratory results; in this case $\xi_{B} \approx 0.03933$ implying that approximately $3.9 \%$ of the frictional energy is expended on attrition of fine material from the surface of 
particles. This is in agreement with the more approximate result (of 4.7\%) obtained from the first parameter study.

In summary, the comparison of PFC3D simulations of shearing-induced attrition with laboratory data, suggest that approximately $2-5 \%$ of the total available frictional work is available for attrition of granular assemblies under shear. This result permits the development of a hybrid strategy for shear-induced secondary fragmentation prediction utilising REBOP simulations (to predict stresses and shear strains within IMZs) and comminution models (to predict the evolution of fragment sizes during transit through an IMZ). This hybrid fragmentation prediction methodology is described in the next section.

\section{$4 \quad$ A hybrid methodology for secondary fragmentation prediction}

A hybrid methodology for prediction of drawpoint fragment size distributions has been devised, drawing upon comminution principles and practise in minerals processing. The methodology, illustrated in Figure 5, is called hybrid because it uses a mixture of numerical (SRM and REBOP) and empirical techniques. The underlying assumption is that a cave mine may be treated as a comminution device and analysed in much the same way as downstream comminution devices (crushers, autogenous grinding (AG) mills, ore passes, etc.). The fragment size-distribution of ore exiting a comminution device is predicted using a relevant comminution model, the inputs to which are:

- the fragment size-distribution of ore entering the comminution device (primary fragmentation)

- the breakage properties of the ore passing through the device

- the effective comminution energy the device imparts on the ore as it passes through the device.

In cave-mining, the mine itself is the comminution device. The first two input parameters above can be estimated using existing techniques. The appropriate input fragment size-distribution for a cave mine is the primary fragmentation size-distribution, which may be estimated using either SRM analysis or BCF. The breakage properties of the ore may be measured in drop weight tests on core samples extracted from the cave (Napier-Munn et. al., 1996). The Shi and Kojovic (2007) breakage model extrapolates small-scale drop weight test results to ores up to $500 \mathrm{~mm}$ in diameter, in a manner consistent with the Hoek-Brown (Hoek and Brown, 1980) criterion. The comminution model algorithms used to simulate the cave process were developed within the JKMRC mine-to-mill projects in base metal, coal mining and processing (McKee and Kojovic, 1997; Esterle et al., 1998).

The third input to the comminution model, the effective comminution energy the device imparts to the ore, is the most problematic to estimate, being a function of the breakage mechanisms the fragments are exposed to during their transit from cave back to drawpoint. As highlighted in the literature survey, three key mechanisms must be considered (impact, shear and compression) and a method devised to estimate the effective comminution energy for each mechanism.

Comminution models offer good potential for estimating secondary fragmentation resulting from fall of caved rock through an air gap. For impact breakage at the air gap, we assume the effective comminution energy is simply the kinetic energy of a body after falling a distance $H$ (the air gap height in metres):

$$
E_{c}{ }^{(\text {impact) }}=\rho g H
$$

where:

$\begin{array}{lll}\rho & = & \text { the rock density }\left(\mathrm{Kg} / \mathrm{m}^{3}\right) . \\ g & = & \text { the acceleration due to gravity }\left(\mathrm{m} / \mathrm{s}^{2}\right) .\end{array}$

It has been widely shown that this formula is appropriate for the prediction of fragmentation sizedistributions obtained from drop weight tests.

For shear-induced fragmentation, we demonstrated in Section 3.3 via comparison of numerical simulation results with the laboratory results of Bridgewater et al. (2003) that an effective measure of shear-induced effective comminution energy is: 


$$
E_{c}^{(\text {shear })}=\xi \sigma \Gamma
$$

where:

$$
\begin{array}{lll}
\sigma & = & \text { the normal stress }(\mathrm{Pa}) . \\
\Gamma & = & \text { the total shear strain applied to a representative volume of ore. }
\end{array}
$$

The constant $(\xi)$ represents the fraction of frictional work available for comminution and is found to lie in the range of $2-5 \%$. We should note however, that the physical origin of, and factors controlling this efficiency factor $(\xi)$, require further investigation.

An explicit measure for compression breakage comminution energy $\left(\mathrm{E}_{\mathrm{c}}{ }^{\text {(compress) }}\right)$ is yet to be devised and will require further research. However, secondary fragmentation in stagnant zones can currently be treated using Hardin's (1985) model. This model accounts for most of the major factors believed to impact secondary fragmentation via compression, it considers both splitting and rounding modes of failure and can be easily incorporated into REBOP. The disadvantage of Hardin's (1985) model is that it does not consider aspect ratio, time or the size distribution resulting from fragmentation. These aspects could be improved through further review of published experiments and fundamental studies on compression breakage of caved rock.

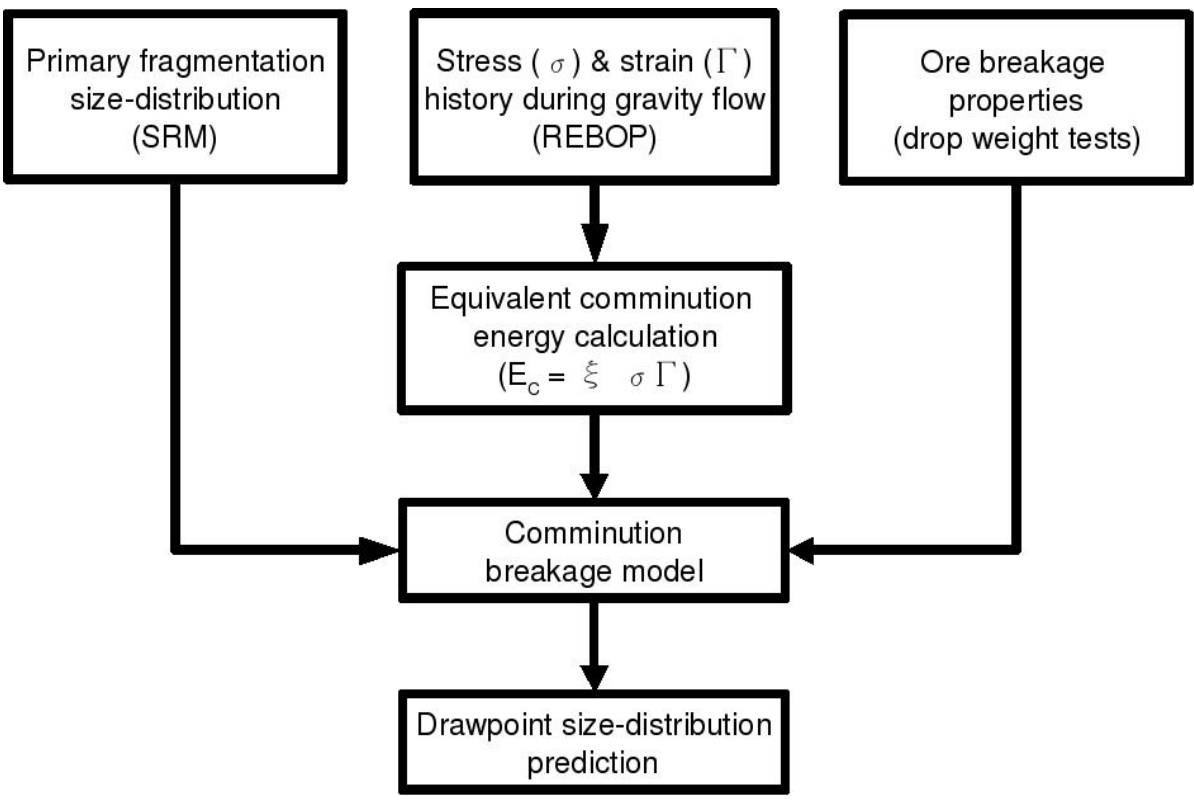

Figure 5 Diagram illustrating the hybrid numerical-empirical methodology for drawpoint sizedistribution prediction

\subsection{Application of the methodology in cave mines}

The hybrid approach was verified at an operational cave mine, firstly considering the change in the draw point fragmentation after the first six months, then 12 months. The stresses and strains estimated using REBOP were used to calculate the effective comminution energies in the movement zones, as noted above. These energies were used to predict the draw point product size distributions. As shown in Figure 6, the drawpoint size-distributions were accurately predicted by the hybrid model when shear-induced comminution efficiency factor of $\xi=5 \%$ was used. This is in agreement with the predictions from the microphysical attrition model discussed in the previous section. Assuming $100 \%$ of the shear-induced energy is used in comminution results in a significantly finer product as illustrated in Figure 6 by the "PRED 1st 6 MTHS (IMZ)" curve. 


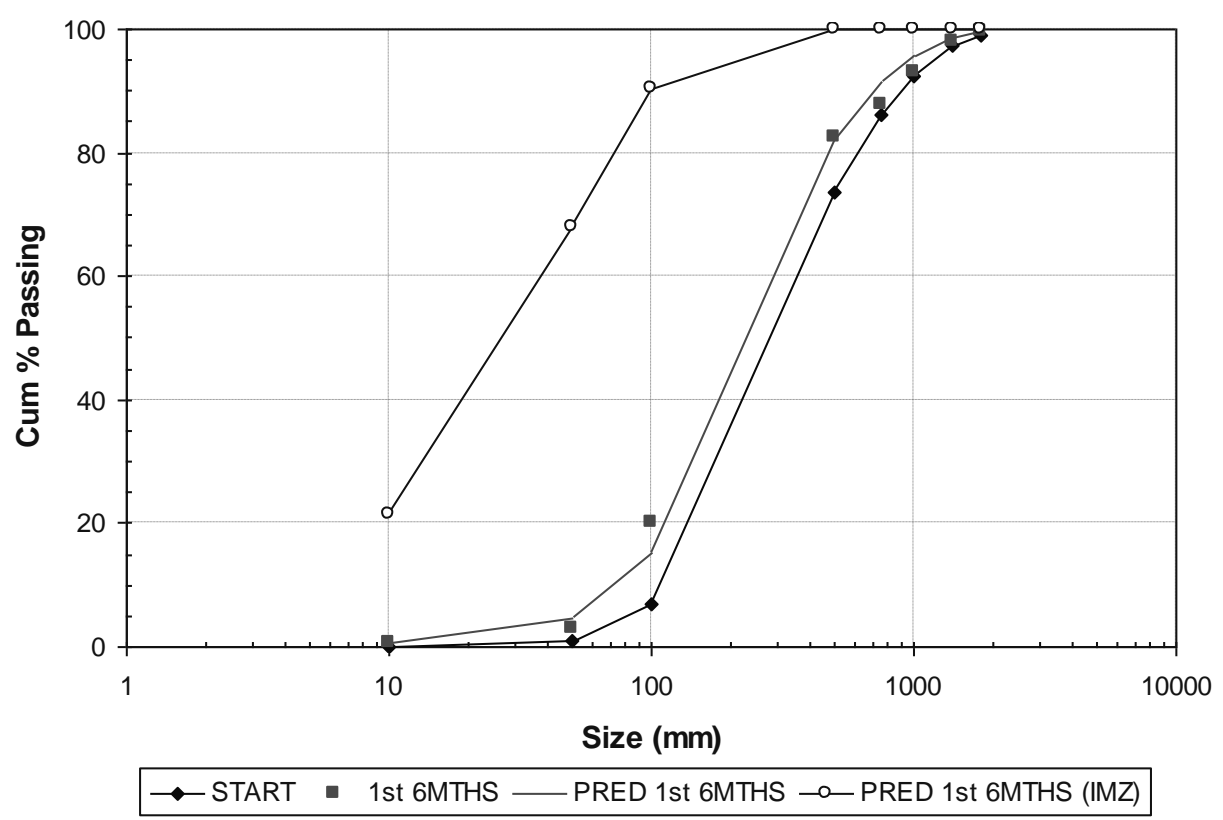

Figure 6 Comparison of actual and predicted drawpoint size-distributions for a cave mine. Good agreement the actual drawpoint size-distribution was obtained when only $5 \%$ of the total shear work in the IMZ was made available for comminution (an independent confirmation of the micromechanical results in Section 3)

Comminution models were then used to simulate the additional size reduction likely to be experienced beyond the drawpoint, considering handling and crushing. Breakage in the hoisting and conveying to the stockpile can be modelled from energy considerations, as shown in the JKMRC mine-to-mill projects. Standard crusher models are suitable for the breakage predictions in the underground gyratory crusher. These simulations provided a fragmentation profile at each of the stages from initial caving to when the ore reports to the semi-autogenous (SAG) mill.

The simulations for this cave mine assumed an underground handling system consisting of a grizzly screen, ore bins, crusher and several conveyor transfer points. Figure 7 shows the change in fragmentation from the SRM to the SAG mill feed, with actual mill feed size data included in the chart. The close agreement in the estimated SAG feed size distribution bodes well for the hybrid approach, which when combined with the suite of comminution models for the handling system, offers a complete 'cave back-to-mill' simulation capability. 


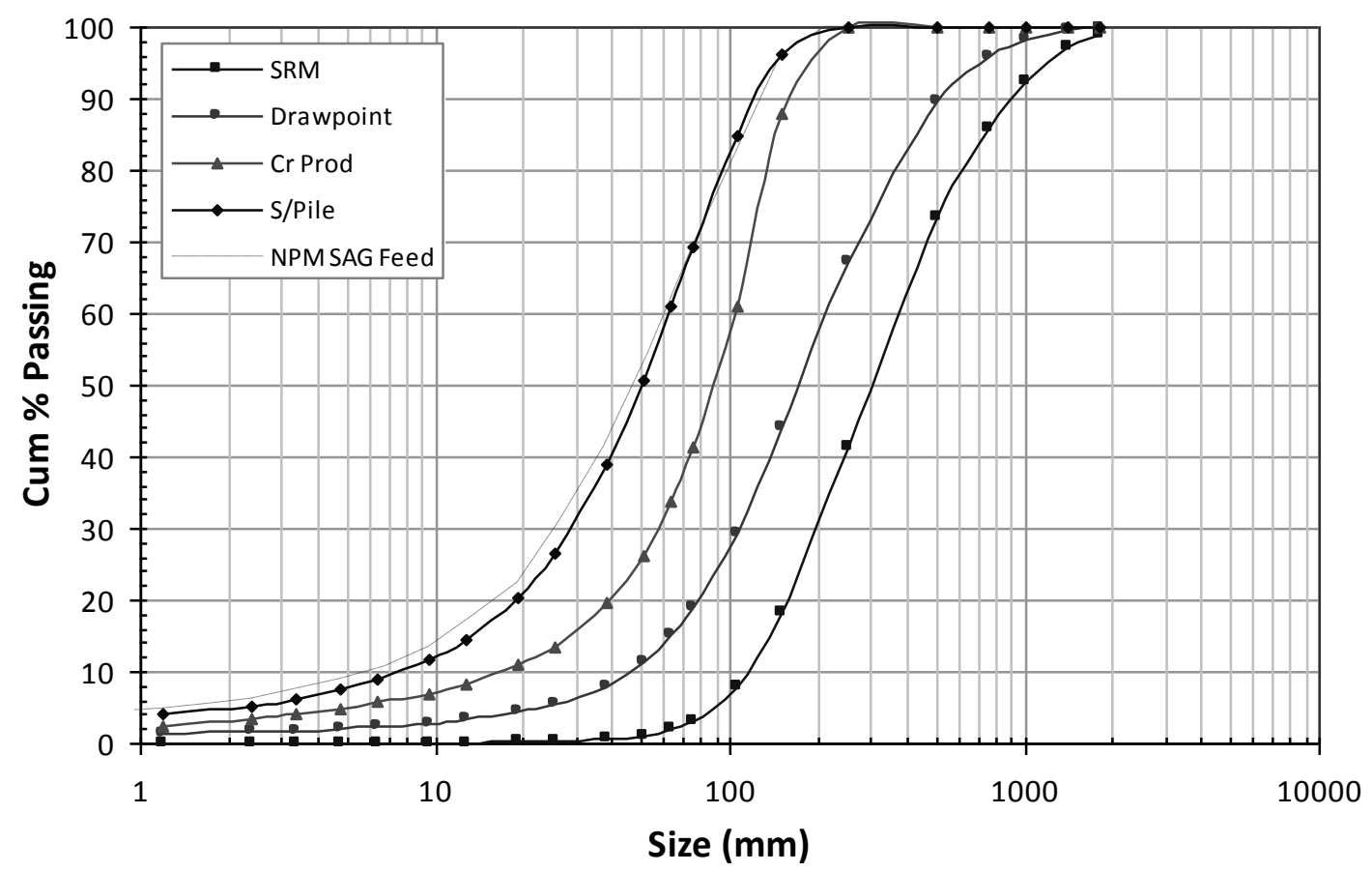

Figure 7 Chart showing the change in fragmentation at a cave mine after 12 months, including the SRM, drawpoint, jaw crusher (Cr Prod) and stockpile (S/Pile); dotted line shows actual SAG feed size distribution from JKMRC surveys

\section{Conclusions}

We have demonstrated a methodology for prediction of secondary fragmentation size-distributions that takes account of impact, shear and compression breakage within cave mines. The methodology relies on inputs from existing numerical techniques for primary fragmentation prediction (the SRM methodology) and estimation of the stresses and strains imposed on caved rock during transit through the mine (via REBOP). Currently we draw upon established minerals processing comminution models to predict the evolution of drawpoint fragment size-distributions. Back-analysis of drawpoint yield data from a cave mine provides confidence that this hybrid methodology can predict drawpoint size-distributions for at least some existing caving operations. There is need for more rigorous validation of the methodology involving both backanalysis of drawpoint data from a range of different caving environments and sensitivity studies to determine the key factors governing size-distribution predictions of the methodology itself.

Central to the proposed methodology are relations governing the product size-distributions for each of the three secondary fragmentation mechanisms: impact, shear and compaction. At this stage, the relations are highly simplified, neglecting rock mass properties (such as joint strengths, orientations and persistence) and shape-dependent effects (such as aspect ratio and impact angles). A suitable relation for predicting compaction-induced fragmentation in stagnant zones is yet to be developed. The back-analysis of drawpoint yield data has highlighted some of the limitations of the approach, in particular the simplified treatment of shear-induced effective comminution energy via the efficiency parameter $(\xi)$. We recommend further backanalyses using drawpoint yield data from existing caving operations to improve knowledge on the applicability and limitations of the hybrid approach.

Further studies using physics-based numerical models (such as PFC3D) are warranted to validate and refine the relationships for predicting evolution of fragment size-distributions during gravity flow in cave mines. With further refinement, we are confident our approach will have utility for the forward prediction of drawpoint fragmentation and become an important tool for the design and operation of future caving operations. 


\section{Acknowledgements}

This research was funded by the Mass-Mining Technologies (MMT) Research Project. The authors gratefully acknowledge the guidance and support of MMT sponsors and research providers. Drawpoint yield data used in the back-analysis was provided by one of the MMT sponsors.

\section{References}

Bridgwater, J., Utsumi, R., Zhang, Z. and Tuladhar, T. (2003) Particle attrition due to shearing - the effects of stress, strain and particle shape, Chemical Engineering Science, Vol. 58, pp. 4649-4665.

Brown, E.T. (2003) Block Caving Geomechanics (The International Caving Study I, 1997-2000) University of Queensland, JKMRC Monograph Series in Mining and Mineral Processing 3, Indooroopilly, Australia.

Castro, R. (2006) Study of the mechanisms of granular flow for block caving, PhD thesis, University of Queensland, Australia.

Coop, M.R., Sorensen, K.K, Bodas Freitas, T. and Georgoutsos, G. (2004) Particle breakage during shearing of a carbonate sand, Geotechnique, Vol. 54, No. 3, pp. 157-163.

Esterhuizen, G.S. (1999) A Program to Predict Block Cave Fragmentation, Technical Reference and User's Guide, Version 3.0.

Esterhuizen, G.S. (1998) ICS Meeting Minutes, BCF Review, Brisbane, Australia.

Esterle, J.S., O'Brien, G.O., Thornton, D., Kojovic, T. and Firth, B. (1998) Tracking coal fragmentation from pit face top plant feed, Mine to Mill 1998 Conference, Brisbane, Australia, pp. 165-169.

Fukumoto, T. (1992) Particle breakage characteristics in granular soils, Soils Foundations, Vol. 32, No. 1, pp. $26-40$.

Ghadiri, M., Ning, Z., Kenter, S.J. and Puik, E. (2000) Attrition of granular solids in a shear cell, Chemical Engineering Science, Vol. 55, pp. 5445-5456.

Hardin, B.O. (1985) Crushing of soil particles, Journal of Geotechnical, Engineering ASCE, Vol. 111(10), pp. 1177-1192.

Hoek, E. and Brown, E.T. (1980) Underground Excavations, In Rock, London, Institution of Mining and Metallurgy.

Lade, P.V. and Yamamuro, J.A. (1996) Undrained sand behavior in axisymmetric tests at high pressures, Journal of Geotechnical Engineering, ASCE, Vol. 122(2), pp. 120-129.

Laubscher, D.H. (2000) Block Caving Manual, prepared for International Caving Study, JKMRC and Itasca Consulting Group Inc., Brisbane, Australia.

Lee, D.M. (1992) The angles of friction of granular fills, PhD dissertation, University of Cambridge.

Lee, K.J. and Farhoomand, I. (1967) Compressibility and crushing of granular soils in anisotropic triaxial compression, Canadian Geotechnical Journal, Ottawa, Canada, Vol. 4(1), pp. 68-86.

Lorig, L.J. and Cundall, P.A. (2000) A rapid gravity flow simulator, Final Report, International Caving Study, E.T. Brown (ed), JKMRC and Itasca Consulting Group Inc., Brisbane, Australia.

Marsal, R.J. (1967) Large scale testing of rockfill materials, Journal of Soil Mecanics and Foundations Division, ASCE, Vol. 93(2), pp. 27-43.

McDowell, G.R. (2005) A physical justification for $\log \mathrm{e}-\log \mathrm{s}$ based on fractal crushing and particle kinematics, Geotechnique, Vol. 55(9), pp. 697-698.

McDowell, G.R. and Bolton, M.D. (1998) On the micromechanics of crushable aggregates, Geotechnique, Vol. 48(5), pp. 667-679.

McDowell, G.R., Bolton, M.D. and Robertson, D. (1996) The fractal crushing of granular materials, Journal of the Mechanics and Physics of Solids, Vol. 44(12), pp. 2079-2102.

McKee, D.J. and Kojovic, T. (1997) Comminution in a mining context, in Proceedings SME'97 Comminution Practices Conference, Denver, Colorado, pp. 271-278.

Nakata, Y., Hyodo, M., Hyde, A.F.L., Kato, Y. and Murata, H. (2001) Microscopic particle crushing and sand subjected to one-dimensional compression, Soils and Foundations, Japanese Geotechnical Society, Vol. 41(1), pp. 69-82.

Napier-Munn, T.J., Morrell, S., Morrison, R.D. and Kojovic, T. (1996) Mineral Comminution Circuits: Their Design and Optimisation, JKMRC, University of Queensland, Brisbane, $413 \mathrm{p}$.

Paramanathan, B.K. and Bridgwater, J. (1983) Attrition of solids-II: Material behaviour and the kinetics of attrition, Chemical Engineering Science, Vol. 38, pp. 207-224.

Pierce, M.E. (2004) Final Report, International Caving Study II, JKMRC and Itasca Consulting Group Inc., Brisbane, Australia.

Pierce, M.E. (2007) Six Monthly Technical Report, Gravity Flow Mechanics and SLC Blasting and Flow, Sub-Project No. 1.2 and 3.2.3: REBOP Development and Disturbed Flow Modelling, Itasca Consulting Group Inc., Report to Mass Mining Technology Project, 2004-2007, ICG06-2292-13-Tasks 1 and 9, March.

Pierce, M., Cundall, P., Mas Ivars, D., Darcel, C., Young, R.P., Reyes-Montes, J. and Pettitt, W. (2006) Six Monthly Technical Report, Caving Mechanics, Sub-Project No. 4.2: Research and Methodology Improvement, and Sub- 
Project 4.3, Case Study Application, Itasca Consulting Group, Inc., Report to Mass Mining Technology Project, 2004-2007, ICG06-2292-1-Tasks-2-3-14, March.

Rumpf, H. (1973) Physical aspects of comminution and a new formulation of a Law of Comminution, Powder Technology, Vol. 7, pp. 145-159.

Shi, F. and Kojovic, T. (2007) Validation of a Model for Impact Breakage Incorporating Particle Size Effect, International Journal of Mineral Processing, Vol. 82, pp. 156-163.

Turcotte, D.L. (1986) Fractals and fragmentation, Journal of Geophysical Research, Vol. 91(B2), pp. 1921-1926.

Vogel, L. and Peukert, W. (2003) Breakage behaviour of different materials - construction of a mastercurve for the breakage probability, Powder Technology, Vol. 129, pp. 101-110.

Vogel, L. and Peukert, W. (2004) Determination of material properties relevant to grinding by practicable labscale milling tests, International Journal of Minerals Processing, Vol. 74S, pp. 329-338.

Weibull, W. (1951) A statistical distribution function of wide applicability, Journal of Applied Mechanics, Vol. 9, pp. 293-297.

Yamamuro, J.A. and Lade, P.V. (1996) Drained sand behavior in axisymmetric tests at high pressures, Journal of Geotechnical Engineering, ASCE, Vol. 122(2), pp. 109-119.

Yamamuro, J.A., Bopp, P.A. and Lade, P.V. (1996) One-dimensional compression of sands at high pressures, Journal of Geotechnical Engineering, ASCE, Vol. 122(2), pp. 147-154.

Yamamuro, J.A. (1993) Instability and behaviour of granular materials at high pressures, PhD dissertation, Deptartment of Civil Engineering, University of California, Los Angeles. 
